Trauma Surgery \& Acute Care Open

\title{
The effect of perturbations of the glycocalyx on microvascular perfusion in the obese trauma population: an in vitro study
}

\author{
Lawrence N Diebel, Alexander Liviu Marinica, David Edelman, David Liberati
}

Surgery, Wayne State University School of Medicine, Detroit, Michigan, USA

Correspondence to Dr Lawrence N Diebel; Idiebel@ med.wayne.edu (c) Author(s) (or their employer(s)) 2021. Re-use permitted under CC BY-NC. No commercial re-use. See rights and permissions. Published by BMJ.

To cite: Diebel $\mathrm{LN}$,

Marinica AL, Edelman D,

et al. Trauma Surg Acute Care Open 2021;6:e00711.

\section{ABSTRACT}

Objectives Patients with morbid obesity have impaired responses to resuscitation following severe injury, which may contribute to adverse outcomes. Obesity is associated with microvascular dysfunction and metabolic changes associated with altered hemorheological profiles. These include decreased red blood cell (RBC) deformity associated with increased aggregation and adhesion. These RBC changes may be impacted by the glycocalyx layer of the endothelial cell (EC) and RBC. Degradation of either or both glycocalyx layers may impair microvascular perfusion. This was studied from blood obtained from patients with obesity and in an in vitro microfluidic device to mimic the microvascular environment.

Methods RBCs were obtained from fresh whole blood from normal controls and patients with obesity (body mass index 37.6-60.0). RBC glycocalyx was indexed by fluorescent intensity and shedding of EC glycocalyx components into the serum was determined by measurement of syndecan-1 and hyaluronic acid. In a second set of experiments, human umbilical vein endothelial cell monolayers (HUVEC) were perfused with RBC suspensions from control and patients with obesity using a microfluidic device and RBC adherence under normoxic or shock conditions (hypoxia+epinephrine) was determined using confocal microscopy. HUVEC glycocalyx thickness and shedding were also measured.

Results Microfluidic studies demonstrated that RBC obtained from subjects with obesity had increased adhesion to the endothelial layer, which was more profound under shock conditions versus normal subjects. This appeared to be related to increased shedding of the endothelial glycocalyx following shock as well as a diminished RBC glycocalyx layer in the obese population. Conclusion Blood from patients with obesity have decreased RBC glycocalyx thickness accompanied by evidence of increased EC glycocalyx shedding. In vitro adhesion to the endothelium was more pronounced with RBC from patients with obesity and was significantly greater under 'shock conditions'. Hemorheological properties of RBC from patients with obesity may account for failure of standard resuscitation procedures in the trauma patient.

\section{LEVEL OF EVIDENCE}

\section{INTRODUCTION}

The impact of the increasing number of individuals suffering from obesity on healthcare in the USA and other industrial countries is well described. As the incidence of obesity in the trauma population mirrors that of the general public, it is apparent that it is of significant interest to trauma care providers. Numerous studies have described the effect of obesity on management and subsequent outcomes in trauma patients. ${ }^{1}$ These studies have strongly suggested that obesity is associated with increased morbidity and mortality following trauma. Both altered responses to traumatic stress as well as existing comorbidities likely contribute to poorer outcomes in these patients. In particular, impaired response to hemorrhagic shock and resuscitation have been shown both clinically and in animal studies. $^{23}$

The microcirculation is a key player in obesityrelated cardiovascular disease. ${ }^{4}$ In addition, hemorheological abnormalities with red blood cells (RBCs) in the patient with obesity have been described..$^{5-7}$ Notably, the importance of the glycocalyx layer of both the endothelium and the RBC is increasingly being recognized. However, the interaction of RBC dynamics and the endothelium in obesity have not been well described, particularly in shock conditions.

We have previously used microfluidic technology to study RBC-endothelial cellular interaction under 'normal' and 'shock' biomimetic conditions. ${ }^{8}$ In the current study, we hypothesize that perturbation of the glycocalyx layers of the RBC and the endothelium would impair microvascular perfusion and have a synergistic effect. This was studied in our in vitro microfluidic-based model.

\section{METHODS}

Human umbilical vein endothelial cells culture Human umbilical vein endothelial cells (HUVEC) were purchased from Lonza Walkersville (Walkersville, Maryland, USA). Cells were grown in a 75 $\mathrm{cm}^{2}$ flask using complete media (EGM-2 BulletKit, Lonza). Time to subculture is $5-9$ days with media changes every 2 days. Cells are subcultured at $85 \%$ confluence using $2 \mathrm{~mL}$ of $0.5 \%$ trypsin-EDTA (Life Technologies, Carlsbad, California, USA). A new culture flask is prepared and the remaining cells are used to seed the microfluidic channels of a BioFlux 48 well plate (Fluxion Bio, San Francisco, California, USA) that has been primed and coated with $100 \mu \mathrm{g} / \mathrm{mL}$ fibronectin (Fisher Scientific) for 1 hour at room temperature. Monolayers were formed within the microfluidic channels after overnight perfusion of the cells with complete media at a shear force of $1 \mathrm{dyne} / \mathrm{cm}^{2}$. HUVEC at passage 4-7 were used for all experiments. 


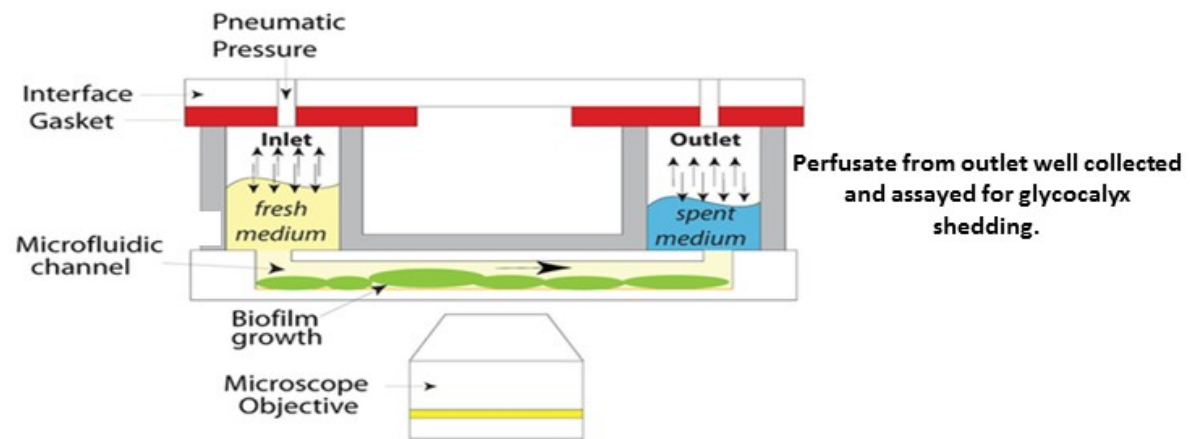

Experimental Overview

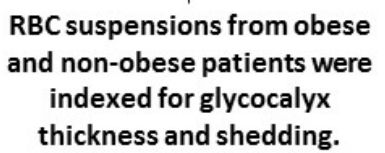

RBC suspensions from obese indexed for glycocalyx thickness and shedding.

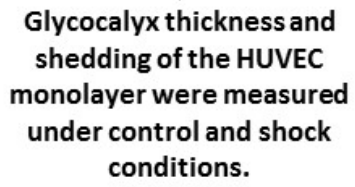

\section{RBC adherence to HUVEC monolayers \pm Hypoxia and epi was determined using confocal microscopy.}

Figure 1 Experimental overview. epi, epinephrine; HUVEC, human umbilical vein endothelial cell; RBC, red blood cell.

Well plate microfluidic device system

The main components of the microfluidic device system (MDS) include BioFlux 48 well plates $\left(4-20 \mathrm{dyn} / \mathrm{cm}^{2}\right)$, a pressure interface device, a controller instrument and software for the instrument control and image analysis (figure 1). The BioFlux plates contain an array of microfluidic flow channels on a well plate format which are connected to inlet and outlet wells. The pressure interface device covers the top of the well plate and applies a controlled pneumatic pressure from the control instrument. This serves to drive the fluid or perfusate through the microfluidic channels at a user-defined flow rate. An observation window in the bottom of the microfluidic channels allows imaging by microscopy. The MDS software allows control of the flow settings as well as other parameters including image analysis. Thus, the microfluidic device allows study of the glycocalyx barrier function under flow-induced shear stress under controlled experimental conditions.

\section{Preparation of red blood cell concentrate}

Whole blood was collected from 6 healthy volunteers (aged 35-55 years) and 11 patients with obesity (aged 33-69 years). Most of the blood samples were obtained from females (81\%). The body mass index in the healthy volunteer group ranged from 19 to $25 \mathrm{~kg} / \mathrm{m}^{2}$ and in the obese group 37.6 to $60 \mathrm{~kg} / \mathrm{m}^{2}$. Patients in the obese group were being evaluated for bariatric surgery in the outpatient setting and all had metabolic syndrome including diabetes and hypertension requiring medication. Blood obtained was centrifuged for $10 \mathrm{~min}$ at $1400 \times \mathrm{g}$. The plasma was collected and stored and the buffy coat discarded. The remaining RBCs were saved and centrifuged two more times to yield isolated RBC. RBC samples were diluted in phosphate-buffered saline (PBS) at $1.5 \%$ for the RBC adhesion assay. This concentration was chosen based on a study by Anniss and Sparrow. ${ }^{9}$

\section{Experimental design}

Once confluent HUVEC monolayers are formed, RBC obtained from volunteers (fresh) or from patients with obesity were added to the perfusate at different flow rates. In some experiments, HUVECs are exposed to $10-3 \mu \mathrm{M}$ epinephrine (epi) and/or hypoxia for $90 \mathrm{~min}$ at $37^{\circ} \mathrm{C}\left(1 \% \mathrm{O}_{2}\right.$ balance nitrogen $)$. Following this, standard culture conditions are reinstated $\left(37^{\circ} \mathrm{C}\right.$ with $5 \%$ $\mathrm{CO}_{2}$; reoxygenation). $\mathrm{RBC}$ adhesion to the endothelial cell monolayer under the microfluidic device under constant flow conditions was determined by light microscopy (figure 1). RBC adherence strength was determined by progressively increasing the shear rate from 0.5 to $5 \mathrm{dyne} / \mathrm{cm}^{2}$. Glycocalyx shedding in the endothelial monolayer and in normal or obese RBCs was assessed by measuring syndecan-1 and hyaluronic acid (HLA) present in cell supernatants. Glycocalyx injury was also assessed in HUVEC and RBC by staining with fluorescein isothiocyanateconjugated wheat germ agglutinin (FITC-WGA, Sigma Aldrich, St. Louis, Missouri, USA) antibody which binds to $N$-acetyl neuraminic acid and $\mathrm{N}$-acetyl glucosamine residues of proteoglycans and glycoproteins present in the glycocalyx and visualizing the glycocalyx using a fluorescent microscope. Image analysis for quantification of glycocalyx thickness was accomplished using Volocity software. Glycocalyx shedding of the endothelial cell monolayer was also indexed by measuring syndecan- 1 and HLA present in cell supernatants.

\section{Syndecan-1 and hyaluronic acid analysis}

Quantitative measurement of syndecan-1 protein and HLA shed by RBC and the endothelial cell monolayer into the supernatants was accomplished using the Syndecan-1 Human ELISA kit (Abcam, Cambridge, Massachusetts, USA) and the Hyaluronic Acid Immunoassay kit from R \& D Systems, Minneapolis, Minnesota, USA, respectively. Standards and unknown samples are added to the microplate wells and assay procedures followed. The optical density is determined using a microplate reader set to $450 \mathrm{~nm}$ and the concentration of syndecan-1 and HLA in the supernatants is calculated using a standard curve. The sensitivity of the syndecan- 1 and HLA ELISAs are 5 and $10 \mathrm{pg} / \mathrm{mL}$, respectively. 
Red blood cell adhesion

RBCs from subjects with and without obesity were added to the perfusate and allowed to flow through the microfluidic device at sequentially increasing shear rates of $0.5-5 \mathrm{dyne} / \mathrm{cm}^{2}$. Flow of the RBC through the microfluidic device at each increasing shear rate was stopped after $5 \mathrm{~min}$ and adherent cells photographed and counted using the Zeiss Observer Spinning Disk Confocal Microscope (Microscopy, Imaging and Cytometry core facility at Wayne State University, Detroit, Michigan, USA). Non-adherent RBCs were removed by washing the monolayer with PBS 2 times (shear rate of $0.5 \mathrm{dyne} / \mathrm{cm}^{2}$ for $1 \mathrm{~min}$ to wash).

\section{Fluorescent imaging and thickness of glycocalyx}

HUVEC were cultured in endothelial cell growth medium supplemented by growth factors (EGM-2 BulletKit) Lonza. Before cell seeding, microchannels were coated with human fibronectin $(100 \mu \mathrm{g} / \mathrm{mL}$, Fisher Scientific) for 1 hour at room temperature. HUVEC suspensions were seeded into the outlet wells of a 48 well BioFlux plate and infused into the microchannel network using the BioFlux 200 system (Fluxion Biosciences). Cells were cultured for 24 hours with complete media under flow conditions (shear force of $1 \mathrm{dyne} / \mathrm{cm}^{2}$ ) or static conditions. Live cell staining was performed inside the microfluidic channels using WGA conjugated with FITC. Briefly, endothelial cell cultures treated with epi+hypoxia/reoxygenation (HR) or standard media had FITC-WGA infused into the microchannel network using the BioFlux 200 system and cells were allowed to incubate for $30 \mathrm{~min}$. The cells were washed 2 times with PBS and fresh culture medium was subsequently added and cells were examined under a fluorescent microscope. Further image analysis was performed using Volocity software at the Microscopy, Imaging and Cytometry core facility at Wayne State University. Measurement of glycocalyx thickness was achieved by XYZ image stacks of the endothelial cell layer using a Leica TCS SP5 microscope and a $20 \times$ objective. In a separate series of experiments, normal and obese RBC glycocalyx thickness and fluorescence intensity measurements of the glycocalyx were also determined. Briefly, $\mathrm{RBC}$ were resuspended and incubated in $\mathrm{PBS} / 1 \%$ bovine serum albumin containing FITC-WGA for $30 \mathrm{~min}$ at $37^{\circ} \mathrm{C}$. The RBCs were subsequently centrifuged $(1400 \times \mathrm{g}$ for $10 \mathrm{~min})$ and the supernatant removed. RBCs were resuspended in PBS and centrifuged two more times to remove excess FITC-WGA antibody. RBCs were subsequently mounted on a slide with coverslip and imaged using a Leica TCS SP5 inverted fluorescent microscope with a $63 \times$ oil objective. Further image analysis was performed using Volocity software at the Microscopy, Imaging and Cytometry core facility at Wayne State University. Measurement of glycocalyx thickness was achieved by XYZ image stacks of the endothelial cell layer using a Leica TCS SP5 microscope and a $20 \times$ objective.

\section{Statistical analysis}

An analysis of variance with a post hoc Tukey's test was used to analyze the data. Statistical significance was inferred at $\mathrm{p}$ values of $<0.05$. All data are expressed as mean \pm SD.

\section{RESULTS}

RBC obtained from normal patients or patients with obesity were stained with FITC-WGA. Figure 2A demonstrates significant differences in the fluorescence intensity of RBC in these groups, with an almost 50\% decrease in the obese group. Shedding of $\mathrm{RBC}$ glycocalyx components is shown in figure $2 \mathrm{~B}$. There was a 2.6-fold increase in syndecan-1 and a 3.2-fold increase in HLA shedding noted in RBC supernatants obtained from subjects with obesity versus subjects without obesity. This corresponds to the difference in RBC glycocalyx thickness.

We then studied the effects of our biomimetic shock conditions on endothelial glycocalyx integrity (thickness) and relative shedding of its glycocalyx components (figure 3A,B). Shock conditions decreased glycocalyx thickness by approximately $50 \%$, the average glycocalyx thickness was one-third of control values. Endothelial glycocalyx shedding was increased $3.6 \times$ for syndecan-1 and $6.6 \times$ for HLA following shock conditions versus control.

Finally, the interaction between RBC and endothelial cells was studied under microfluidic flow conditions (figure 4). There was a 3.2-fold increase in RBC adhesion with RBC obtained from patients with obesity versus patients without obesity. After exposure of endothelial monolayers to shock conditions, there was no increase in RBC vascular adherence in the non-obese population. However, there was a 1.5 -fold increase in vascular adhesion with RBC obtained from patients with obesity following exposure to HR and epi.

\section{DISCUSSION}

Obesity is associated with structural and functional changes in the microvasculature, which leads to impaired organ perfusion
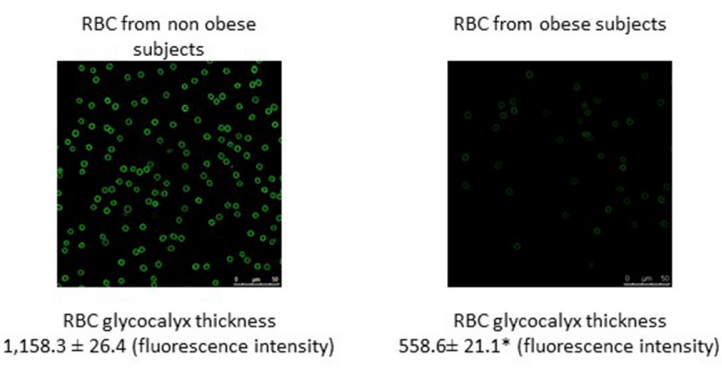

A

${ }^{*} p<0.05$ vs. RBC thickness from non obese subjects.

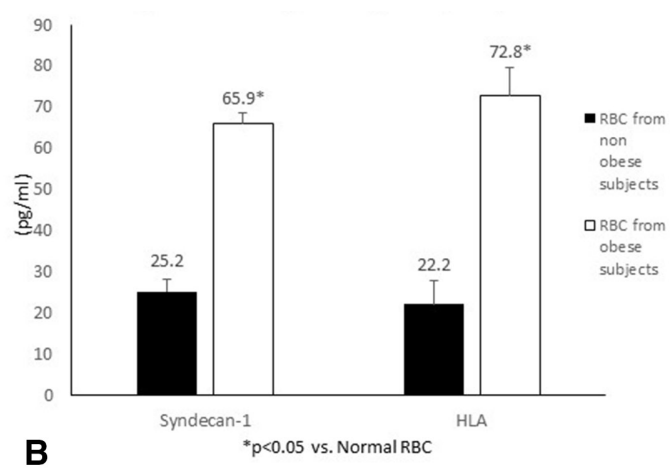

Figure 2 (A) Red blood cell (RBC) glycocalyx staining and fluorescence intensity measurement. RBC glycocalyx from patients with and without obesity was stained with fluorescein isothiocyanate-conjugated wheat germ agglutinin antibody after exposure to hypoxia/reoxygenation and epinephrine. Pictures were taken with a Leica TCS SP5 inverted fluorescent microscope and fluorescent intensity measurements were taken. (B) Shedding of RBC glycocalyx components. ELISA kits were used to quantitate syndecan-1 and hyaluronic acid levels present in RBC from patients with and without obesity exposed to hypoxia $\left(1 \% \mathrm{O}_{2}\right)$ and epinephrine perfusion for $90 \mathrm{~min}$. 


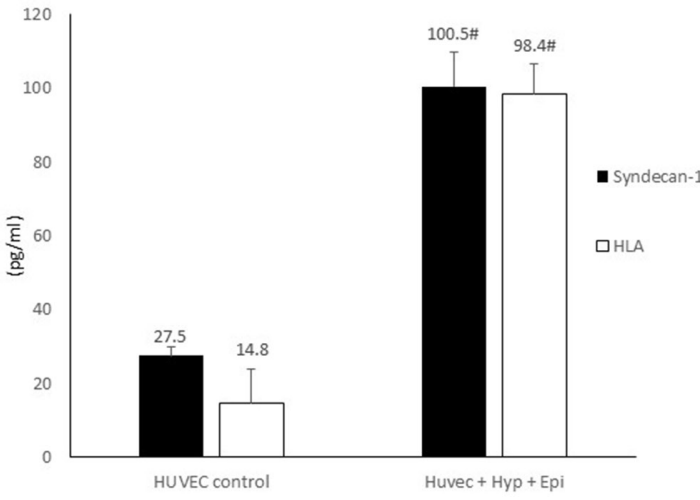

A

\#p $<0.05$ vs. HUVEC control

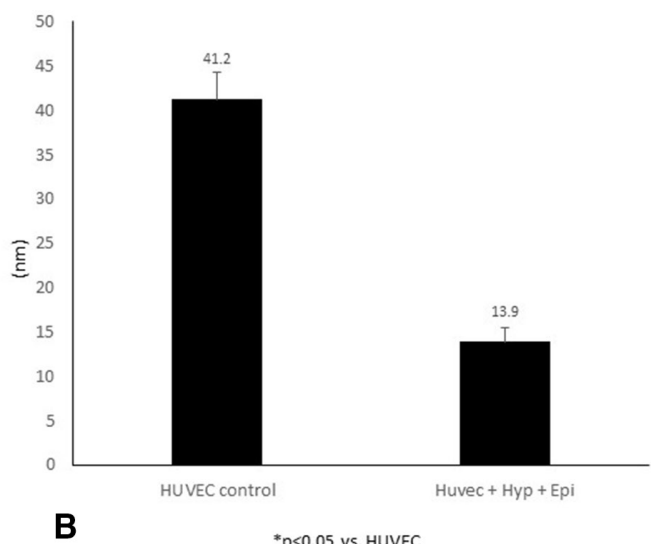

Figure 3 (A) Glycocalyx shedding in human umbilical vein endothelial cell (HUVEC) monolayers. ELISA kits were used to quantitate syndecan-1 and hyaluronic acid (HLA) levels present in the perfusate collected from HUVEC exposed to hypoxia (Hyp) $\left(1 \% \mathrm{O}_{2}\right)$ and epinephrine (epi) perfusion for 90 min. (B) HUVEC glycocalyx thickness measurement. Three-dimensional XYZ image stacks were acquired and processed and analyzed using Volocity cellular imaging and analysis software to assess glycocalyx thickness.

and resultant dysfunction. ${ }^{10}$ Hemorrhagic shock is associated with impaired tissue perfusion; it is likely therefore that obesity exacerbates perfusion abnormalities associated with shock. A previous study by our group has indeed demonstrated this in an in vitro model of the microcirculation using microfluidic technology. ${ }^{11}$

A key component of the vascular endothelial barrier is the glycocalyx layer. Its functions include mechanotransduction and regulation of the endothelial cell signaling pathway related to angiogenesis, vasoregulation and inflammation. Endothelial glycocalyx degradation has been noted to occur following shock states, including sepsis and trauma. ${ }^{12}$ Lee et al ${ }^{13}$ have also demonstrated that obesity-related impairment in microvascular perfusion is associated with glycocalyx damage in a large epidemiological study.

RBCs are the most abundant cell type in blood and therefore have significant impact on the fluidity of blood under both normal and pathological conditions. ${ }^{5-7}$ Disturbances in $\mathrm{RBC}$ flow properties have been described in the obese patient population. These include increased aggregation and impaired deformation as well as increased adhesion to other blood cell types and to the vascular endothelium which all may impede blood flow. ${ }^{14}$ The etiology of the microrheological distribution of RBC in obesity is uncertain. RBCs have an under-recognized glycocalyx on their surface. During their 120-day life span in circulation, human RBC undergoes several physiochemical

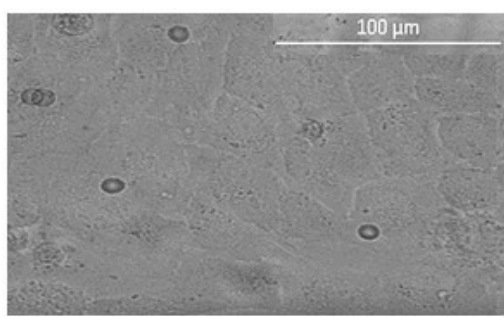

Non obese RBC $62 \pm 12\left(\mathrm{~mm}^{2}\right)$

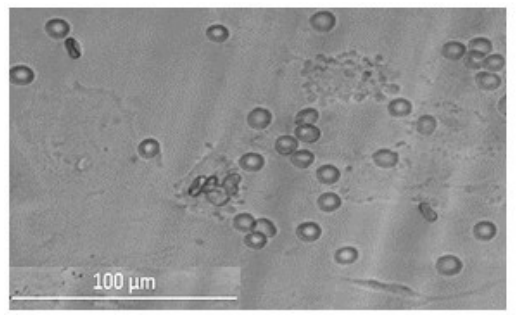

Obese RBC

$201 \pm 35\left(\mathrm{~mm}^{2}\right)^{*}$

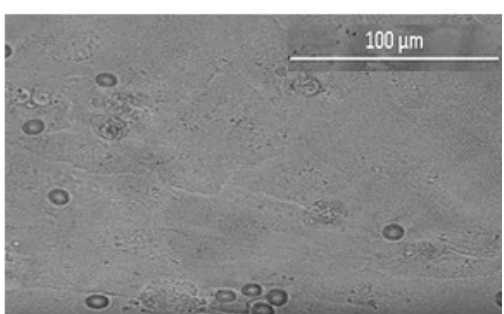

Non obese RBC + Hyp + Epi $70 \pm 15\left(\mathrm{~mm}^{2}\right)$

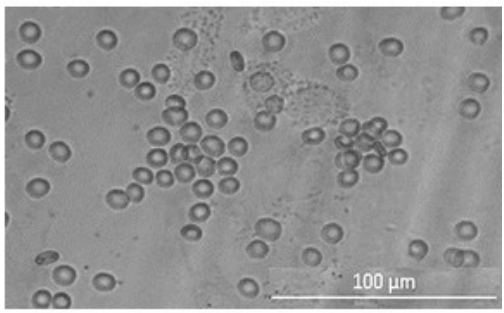

$$
\text { Obese RBC + Hyp + Epi }
$$$$
297 \pm 42\left(\mathrm{~mm}^{2}\right)^{*} \#
$$

Figure 4 Red blood cell (RBC) adhesion to human umbilical vein endothelial cell (HUVEC) from subjects with and without obesity. RBCs from patients without obesity and RBCs from patients with obesity were added to the perfusate and allowed to flow through the microfluidic device. Adherent cells were photographed and counted using the Zeiss Observer Spinning Disk Confocal Microscope (Microscopy, Imaging and Cytometry core facility at Wayne State University, Detroit, Michigan, USA). ${ }^{*}<<0.05$ vs non-obese $R B C, \# p<0.05$ vs same group no hypoxia (Hyp)+epinephrine (epi)). 
changes including a progressive loss of its glycocalyx layer. ${ }^{15}$ We have previously shown the importance of the RBC glycocalyx in cellular adhesion to the vascular endothelium in an in vitro study. ${ }^{8}$ We found that loss of the RBC glycocalyx increased with duration of time of RBC blood bank storage in this study. As it relates to the current study, the laboratory of Schmid-Schobein has demonstrated that proteolytic cleavage of the RBC glycocalyx is a complication of obesity-related metabolic syndrome and may contribute to abnormal microvascular flow properties. ${ }^{16}$ These data suggest that the RBC glycocalyx is important in the rheological properties of blood especially in conditions with altered physiology.

To date, the effect of shared loss of the glycocalyx layer in both RBC and microvascular endothelial cells has not been studied. In our model, we demonstrate a significant increase of RBC adhesion to vascular endothelial cells in studies of blood obtained from patients with obesity. Biomimetic shock conditions in our model led to endothelial glycocalyx degradation and endothelial injury and resultant greater vascular adhesion in only the RBC obtained from subjects with obesity. The biomimetic shock conditions used in our study have been used by us in previous studies. ${ }^{10}$ These include hypoxia-reoxygenation and catecholamine excess, both of which may contribute to endothelial and glycocalyx damage in vivo. ${ }^{17} 18$

Although a number of disturbances in RBC rheology has been demonstrated in obesity, probably the most important one is vascular adhesion as it has been linked to various vascular disorders including sickle-cell anemia, diabetes and hypertension. Notably, these rheological abnormalities are likely co-contributors to perfusion abnormalities in the obese population. In addition, it has been shown when damage occurs in either the glycocalyx of RBC or the EC, the resultant defect further enhances itself through the interaction of these cells. ${ }^{19}$ Nonetheless, our study demonstrates the importance of the glycocalyx layer at the surface of both RBC and the endothelium in the microvasculature under normal and shock conditions. Collectively, the results of the current study and our previous work suggests a need to develop point-of-care technology to assess the integrity of the glycocalyx layer in the clinical arena.

The mechanism(s) responsible for the diminished RBC glycocalyx in samples obtained from subjects with obesity is uncertain. Previous work by us and as well others has shown that oxidative stress and blood storage diminishes the RBC glycocalyx. Increased oxidative stress and a pro-inflammatory state described in obesity are therefore likely important contributors. This information suggests a need to study the effect of blood storage duration on RBC transfusions in the patient with obesity with significant blood loss. Perhaps blood stored $<14$ days or alternative transfusion strategies may be preferable in the critically ill or injured patient with obesity. ${ }^{20}$ Furthermore, as it relates to the RBC storage lesion, a variable that has not been investigated is specific blood donor variability such as age, and comorbidities such as hypertension, diabetes and obesity that may affect RBC component quality from these donors. ${ }^{21-23}$

There are a number of limitations of our study. First, our study involved a small sample size of patients with obesity scheduled for bariatric surgery. We did not control for demographics including age, biological gender or associated comorbidities. Second, we used HUVEC to model the microcirculation in our microfluidic platform, which may not represent other important regional beds including the lungs and viscera. The HUVEC cell line was used to study glycocalyx properties in our study. This cell line was chosen as it has a well-defined thickness under in vitro flow conditions and ex vivo. ${ }^{24}$ Previous studies have shown variable thickness of the glycocalyx layer of established microvascular endothelial cells. ${ }^{25}$ However, these studies were not performed under flow conditions, a prerequisite for the development of a hydrodynamically relevant glycocalyx layer. There is also a growing interest in the interaction of $\mathrm{RBC}$ with other cell types including endothelial cells in the bloodstream. ${ }^{26}$ In this regard, there are data that physical interaction between blood and the endothelium may affect the relative properties of the glycocalyx on both RBC and the endothelium. ${ }^{19}$ In a previous study, we demonstrated a pro-inflammatory phenotype in plasma from subjects with obesity that increased endothelial glycocalyx degradation using our microfluidic platform. Additional studies with RBC and plasma or whole blood studies may further delineate the adverse effects of obesity on the microvasculature in our model.

Finally, we also used a simplistic design for our microchannels and our perfusate consisted of a low hemorheological solution, which may likely underestimate viscosity-related issues in the microvasculature in vivo. The endothelial glycocalyx is now recognized as a critical factor in shock outcome and a target for protecting the microvasculature in trauma. The microfluidic platform may be useful for future studies. ${ }^{27} 28$

Contributors LND, DL, ALM and DE made substantial contributions to the conception or design of the work and also conceived and designed the experiments. LND, DL, ALM and DE drafted the manuscript. ALM, DE, LND and DL critically revised the manuscript for intellectual content. All authors provided final approval of the version to be published.

Funding Funding was provided through departmental sources.

Competing interests None declared.

Patient consent for publication Not required.

Ethics approval Ethics approval was obtained from Wayne State University Institutional Review Board (approval number 1908002437) and participants signed informed consent before inclusion.

Provenance and peer review Not commissioned; externally peer reviewed. Data availability statement All data relevant to study are included in the article.

Open access This is an open access article distributed in accordance with the Creative Commons Attribution Non Commercial (CC BY-NC 4.0) license, which permits others to distribute, remix, adapt, build upon this work non-commercially, and license their derivative works on different terms, provided the original work is properly cited, appropriate credit is given, any changes made indicated, and the use is non-commercial. See: http://creativecommons.org/licenses/by-nc/4.0/.

\section{REFERENCES}

1 Liu T, Chen J-jun, Bai X-jun, Zheng G-shou, Gao W. The effect of obesity on outcomes in trauma patients: a meta-analysis. Injury 2013;44:1145-52.

2 Nelson J, Billeter AT, Seifert B, Neuhaus V, Trentz O, Hofer CK, Turina M. Obese trauma patients are at increased risk of early hypovolemic shock: a retrospective cohort analysis of 1,084 severely injured patients. Crit Care 2012;16:R77.

3 Mittwede PN, Clemmer JS, Bergin PF, Xiang L. Obesity and critical illness: insights from animal models. Shock 2016;45:349-58.

4 Sorop 0, Olver TD, van de Wouw J, Heinonen I, van Duin RW, Duncker DJ, Merkus D. The microcirculation: a key player in obesity-associated cardiovascular disease. Cardiovasc Res 2017;113:1035-45.

5 Baskurt OK, Meiselman HJ. RBC Aggregation: More Important than RBC Adhesion to Endothelial Cells as a Determinant of In Vivo Blood Flow in Health and Disease. Microcirculation 2008;15:585-90.

6 Yedgar S, Kaul DK, Barshtein G. Rbc adhesion to vascular endothelial cells: more potent than RBC aggregation in inducing circulatory disorders. Microcirculation 2008; 15:581-3.

7 Zeng NF, Mancuso JE, Zivkovic AM, Smilowitz JT, Ristenpart WD. Red blood cells from individuals with abdominal obesity or metabolic abnormalities exhibit less deformability upon entering a constriction. PLoS One 2016;11:e0156070.

8 Diebel LN, Liberati DM. Red blood cell storage and adhesion to vascular endothelium under normal or stress conditions: an in vitro microfluidic study. J Trauma Acute Care Surg 2019;86:943-51.

9 Anniss AM, Sparrow RL. Variable adhesion of different red blood cell products to activated vascular endothelium under flow conditions. Am J Hematol 2007;82:439-45. 
10 Eskens BJM, Leurgans TM, Vink H, VanTeeffelen JWGE. Early impairment of skeletal muscle endothelial glycocalyx barrier properties in diet-induced obesity in mice. Physiol Rep 2014;2:e00194.

11 Diebel LN, Peraino N, Westrick J, Shinki K, Liberati DM. Obesity and impaired barrier function after shock: a biomimetic in vitro model using microfluidics. J Trauma Acute Care Surg 2020;89:544-50.

12 Johansson P, Stensballe J, Ostrowski S. Shock induced endotheliopathy (SHINE) in acute critical illness - a unifying pathophysiologic mechanism. Crit Care 2017;21:25.

13 Lee DH, Dane MJC, van den Berg BM, Boels MGS, van Teeffelen JW, de Mutsert $\mathrm{R}$, Heijer Mden, Rosendaal FR, van der Vlag J, van Zonneveld AJ, et al. Deeper penetration of erythrocytes into the endothelial glycocalyx is associated with impaired microvascular perfusion. PLoS One 2014;9:e96477.

14 Pretini V, Koenen MH, Kaestner L, Fens MHAM, Schiffelers RM, Bartels M, Van Wijk R. Red blood cells: chasing interactions. Front Physiol 2019;10:945.

15 Neu B, Sowemimo-Coker SO, Meiselman HJ. Cell-Cell affinity of senescent human erythrocytes. Biophys J 2003:85:75-84.

16 Mazor R, Schmid-Schönbein GW. Proteolytic receptor cleavage in the pathogenesis of blood rheology and co-morbidities in metabolic syndrome. Early forms of autodigestion. Biorheology 2015;52:337-52.

17 Jackson-Weaver O, Friedman JK, Rodriguez LA, Hoof MA, Drury RH, Packer JT, Smith A, Guidry C, Duchesne JC. Hypoxia/Reoxygenation decreases endothelial glycocalyx via reactive oxygen species and calcium signaling in a cellular model for shock. J Trauma Acute Care Surg 2019:87:1070-6.

18 Johansson PI, Ostrowski SR. Acute coagulopathy of trauma: balancing progressive catecholamine induced endothelial activation and damage by fluid phase anticoagulation. Med Hypotheses 2010;75:564-7.
19 Oberleithner H. Vascular endothelium leaves fingerprints on the surface of erythrocytes. Pflugers Arch 2013;465:1451-8.

20 McCully BH, Underwood SJ, Kiraly L, Holcomb JB, Robinson BRH, Minei JP, Stewart RM, Cotton BA, Gordon NT, Martin DT, et al. The effects of cryopreserved red blood cell transfusion on tissue oxygenation in obese trauma patients. J Trauma Acute Care Surg 2018;84:104-11.

21 Sparrow RL. Red blood cell components: time to revisit the sources of variability. Blood Transfus 2017:15:116-25.

22 Murphy EL, Schlumpf K, Wright DJ, Cable R, Roback J, Sacher R, Busch MP,. NHLB Retrovirus Epidemiology Donor Study II. Bmi and obesity in US blood donors: a potential public health role for the blood centre. Public Health Nutr 2012;15:964-71.

23 Vayá A, Suescun M, Solá E, Romagnoli M, Hernández-Mijares A. Rheological blood behaviour is not related to gender in morbidly obese subjects. Clin Hemorheol Microcirc 2012;50:227-9.

24 Chappell D, Jacob M, Paul O, Rehm M, Welsch U, Stoeckelhuber M, Conzen P, Becker BF. The glycocalyx of the human umbilical vein endothelial cell: an impressive structure ex vivo but not in culture. Circ Res 2009;104:1313-7.

25 Janczyk P, Hansen S, Bahramsoltani M, Plendl J. The glycocalyx of human, bovine and murine microvascular endothelial cells cultured in vitro. J Electron Microsc 2010:59:291-8.

26 Pretini V, Koenen MH, Kaestner L, Fens MHAM, Schiffelers RM, Bartels M, Van Wijk R. Red blood cells: chasing interactions. Front Physiol 2019;10:1-17.

27 Tuma M, Canestrini S, Alwahab Z, Marshall J. Trauma and endothelial glycocalyx: the microcirculation helmet? Shock 2016:46:352-7.

28 Kozar RA, Peng Z, Zhang R, Holcomb JB, Pati S, Park P, Ko TC, Paredes A. Plasma restoration of endothelial glycocalyx in a rodent model of hemorrhagic shock. Anesth Analg 2011;112:1289-95. 